\title{
Condiciones de vida y psicosociales de niños migrantes en el noroeste de México
}

\author{
Life and psychosocial terms of migrant children \\ in northwest Mexico
}

\author{
José Ángel Vera Noriega* \\ Jesús Alfonso Robles Luján**
}

\begin{abstract}
Resumen: Con el objetivo de comparar el estado de salud mental de niños migrantes y sedentarios, se estudio a una población de 358 niños migrantes del estado de Guerrero entre 9 y 13 años que viajaban con sus padres a los campos agrícolas tecnificados de riego del noroeste de México y se comparo con 150 niños pareados vecinos y amigos sedentarios residentes de la zona del municipio de Acatlán, Guerrero, México. Las medidas fueron: escala de ansiedad manifiesta para niños - revisada, cuestionario de depresión para niños, y el inventario de estrés infantil. Los resultados del análisis factorial muestran dimensiones consistentes y con pesos factoriales mayores a .35. El 20\% de los menores migrantes presentaba ansiedad, 10.6\% mostraba depresión, y $41.3 \%$ manifestaba niveles de estrés por encima del rango saludable. Los análisis comparativos muestran que los niños migrantes presentan mayores riesgos de padecer ansiedad, depresión y estrés.
\end{abstract}

Palabras-clave: Niños migrantes y sedentarios; Salud mental; Ansiedad; Depresión; Estrés

Abstract: In order to compare the mental health of migrant and sedentary children, the population was defined by 358 migrants children from the state of Guerrero between 9 to 13 years traveling with her parents to technology-intensive irrigation fields crops in northwest Mexico and compared with 150 children sedentary matched with its neighbors and friends, residents of the municipality of Acatlán, Guerrero, Mexico. The measurements were: anxiety scale for children - revised, depression questionnaire for children, and the inventory of childhood stress. The results of factor analysis are consistent dimensions and factor weights greater than .35. Findings indicate that $20 \%$ of migrant children had anxiety, $10.6 \%$ had depression, and $41.3 \%$ reported stress levels above the healthy range. The comparative analysis shows that migrant children are at greater risk of suffering from anxiety, depression and stress.

Keywords: Migrant and sedentary children; Mental health; Anxiety; Depression; Stress

* Investigador Titula del Centro de Investigación en Alimentación y Desarrollo, A. C., México. <avera@ciad.mx>.

**Investigador del Centro de Investigación en Alimentación y Desarrollo, A.C., México. $<$ jejusrobles1981@yahoo.com.mx>.

\begin{tabular}{|c|c|c|c|c|c|}
\hline Civitas & Porto Alegre & v. 10 & n. 2 & p. 345-365 & maio-ago. 2010 \\
\hline
\end{tabular}




\section{Introducción}

La migración ha sido analizada generalmente bajo las miradas de la economía, la demografía, el derecho, la estadística y el empleo, pero solo en escasas ocasiones estos trabajos han sido dirigidos al estudio del los riesgos y vulnerabilidades a los que están expuestos los migrantes y sus familias, así como a las repercusiones que tiene la migración en su calidad de vida y salud. Los trabajadores agrícolas pertenecen a un sector de la población excluida socialmente y marcada por la pobreza. También son abandonados por parte de las investigaciones sociales. Es necesario resaltar que este grupo social está vulnerable a padecer los principales problemas de salud que aquejan a las personas en México.

La migración interna de menores trae consigo pérdidas que les provocan un proceso de duelo, que sumado a los estresores a los que están expuestos, pueden aumentar su vulnerabilidad física y psicológica como consecuencia de los cambios que el niño y la niña enfrentan en el contexto de socialización, alimentación, enfermedad, uso del espacio, rutina de higiene, sueño-vigilia y sobre todo los afectos, emociones, afiliaciones y expectativas.

El problema se plantea desde la atmosfera del trabajo agrícola en los campos agrícolas de uva y hortalizas en el Noroeste de México en la cual se analiza las peculiaridades del niño migrante indígena y sus circunstancias socioeconómicas, sanitarias e higiénicas, de socialización y salud mental. El planteamiento teórico documenta las tres líneas de estudio que se han utilizado para examinar la relación entre migración y salud mental, incluyendo elementos como estresores psicosociales, pérdidas y duelos migratorios, costos y aspectos compensatorios de la migración, y sus sistemas de protección.

\section{Migración interna: expulsores y receptores}

La migración en México es de carácter interno y externo, los parámetros motivacionales, la estructura de las redes sociales de recepción y los mecanismos a través de los cuales el mercado de trabajo hace posible el tráfico de mano de obra es sustancialmente diferente para los dos tipos de itinerancia.

El Fondo de las Naciones Unidas para la Infancia estima que por año, unos 300,000 niños y niñas migran a otros estados de México a buscar trabajo, en ocasiones junto con sus familias, porque muchos de ellos provienen de poblaciones indígenas y se incorporan a actividades laborales en el campo en el norte del país (UNICEF, 2007). Éstos obtienen ciertas pérdidas y ganancias de los cambios que han sufrido por su condición de migrantes en un mundo en proceso de globalización. Dentro de estos cambios podemos identificar la 
pérdida de sus vestimentas tradicionales, desplazadas por una más moderna. Por otra parte, dentro de las ganancias que experimentan se encuentran una mayor disponibilidad de información, teniendo más accesibilidad a medios de comunicación masiva como la televisión por cable, y en algunos casos la computadora, el internet y los juegos de video (Cos, 2000). Gran parte de las familias que migran al norte son de tradición campesina y cuentan con tierras de cultivo de temporal en sus comunidades de origen, pero viven condicionados por los periodos de siembra y cultivo; estimándose que un $70 \%$ de estos campesinos son pertenecientes a municipios indígenas, predominantemente de la mixteca oaxaqueña, valles centrales de Oaxaca, y la sierra guerrerense (Vera, Rodríguez y Borja, 2005).

En el Foro Nacional para la Vida y el Trabajo realizado por el Instituto Nacional para la Educación de los Adultos, se presentó el dato de SEDESOL (2006) según el cual, de los 1.2 millones de jornaleros agrícolas migrantes, $36 \%$ eran hablantes de alguna lengua indígena. De estos, zapotecos, mixtecos, náhuatles y amusgos tenían un predominio numérico (INEA, 2007). Según cifras del Censo de Población y Vivienda de 1995 la concentración de hablantes de lenguas indígenas en el Estado de Sonora, al norte de México y frontera con Estados Unidos de América, sería en el siguiente orden:

\section{Tabla 1: Concentración de Hablantes de Lenguas Indígenas en Sonora según cifras del Censo de Población y Vivienda de 1995 (Faltan datos de hablantes de cucapá y pápago en Sonora)}

\begin{tabular}{lcllc}
\hline \multicolumn{1}{c}{ Grupo étnico } & $\mathbf{N}^{\mathbf{0}}$ de hablantes & & Grupo étnico & $\mathbf{N}^{\mathbf{0}}$ de hablantes \\
${$\cline { 1 - 4 }$} }$ & 36,803 & & Seri & 560 \\
Yaqui & 14,447 & & Triqui & 551 \\
Mixteco & 1,319 & & Tarahumara & 540 \\
Guarijio & 1,127 & & Náhuatl & 531 \\
Zapotec & 738 & & Pima & 451 \\
Maya & 680 & & \\
\cline { 1 - 3 } & & & &
\end{tabular}

Fuente: INEGI, XI Censo General de Población y Vivienda, 1990.

En estos datos, se puede notar como es que lenguas ajenas a las nativas del territorio sonorense igualan o superan en número a las originarias para el censo de ese año.

\section{Los arreglos ambientales en los Estados receptores}

Uno de los principales problemas al momento de la migración hacia el norte del país son las malas condiciones físicas y mecánicas de los vehículos, 
sin recursos para alimentos en traslados que pueden durar hasta tres días de camino (Vera, Rodríguez y Borja, 2005).

A su llegada, se enfrentan con dificultades como las concernientes al equipamiento en los pabellones de descanso en los campos agrícolas, ya que éstos solo en ocasiones cuentan con los servicios necesarios como agua potable (30\%), letrinas (20\%), electricidad (82\%), lugar adecuado para cocinar (36\%), disponibilidad de alimentos accesibles al bolsillo del trabajador migrante, entre otros. Otras de las secciones con las que cuentan las instalaciones para las familias migrantes en los campos, son baños, lavaderos y tendederos comunitarios, camiones o remolques adaptados como aulas escolares, y los más aventajados pueden tener incluso alguna construcción destinada a servir de centro de salud, guardería, salones de clase, entre otros (Vera, Rodríguez y Borja 2005).

La familia completa trabaja sin contrato, de manera discontinua, sin prestaciones de salud, vivienda o educación. Además no existe voluntad por parte de los propietarios ni organizaciones, para darle una solución real y palpable a esta problemática (INEA, 2007; Lara, 1997; Vera, 2005).

Los jornaleros no tienen una organización formal que se pronuncie por la solución de los derechos laborales y defienda sus salarios o condiciones de trabajo, por lo que acuden a organizaciones informales o redes de apoyo familiar, amigos o personas pertenecientes a su entorno étnico o filial. Esta situación, junto con la falta de información y de capacidad analítica, le han dado a los patrones la libertad de omitir algunas atenciones necesarias dirigidas a darles un trato digno como sus empleados (Vera, Rodríguez y Borja, 2005).

\section{Los niños jornaleros agrícolas migrantes}

El Sistema para el Desarrollo Integral de la Familia en el Estado de Sonora, advierte que el $15 \%$ de los niños y niñas en edades entre 7 y 12 años, de una muestra de 2, 280 personas, trabaja en el campo junto con la población adulta (DIF Sonora, 1997).

Por otra parte, la Secretaría de Desarrollo Social asevera que en el 2006, el $20 \%$ de la fuerza de trabajo de los jornaleros agrícolas en México corresponde a población infantil y adolescente entre 6 y 14 años de edad (INEA, 2007). Siendo precisamente el $40 \%$ de esta población en este mismo rango de edad la que presenta retrasos importantes en lectoescritura, debido a que las largas jornadas laborales compiten con los estudios en horarios y energía invertida. De estos niños y niñas, el $64 \%$ de la población mayor a los 12 años no ha terminado la primaria, en parte debido a las altas tasas de interrupción de los ciclos escolares por los de la siembra (Cos, 2000). 
$\mathrm{Al} 42 \%$ de los niños y niñas migrantes se le ha detectado algún estado de desnutrición, que se comparte con problemas de rutina y preparación de alimentos (UNICEF, 2007). Su alimentación en los campos agrícolas, según un estudio llevado a cabo en el Valle de San Quintín, Baja California, consiste básicamente en tacos de papa, frijol, tortilla con sal, tomates, refrescos, naranjadas y café (Cos, 2000).

La formación psicosocial del niño y la niña incorpora el trabajo con los padres en las labores de recolección de frutos y empaque a partir de los 9 años, impidiendo por un lado utilizar el espacio y el tiempo para jugar y por otro, obstaculizando la posibilidad de crear o mantener amistades por su propia itinerancia. Desde la niñez temprana viven en un ambiente de responsabilidades propias de las personas adultas, sufriendo un proceso de desgaste físico por el estrés que el trabajo arduo les genera, y el psicológico por la falta de amigos y el poco tiempo libre que tienen para jugar con sus pares, además de las injusticias asociadas a la clandestinidad de su trabajo, siendo algunos de los principales factores que les impiden vivir una vida saludable para su edad (López, 2002). Esta situación, afortunadamente ha cambiado en algunos campos que se han insertado dentro de las nuevas modalidades de certificación social (Vera, Rodríguez y Borja 2005).

\section{Desarrollo del planteamiento teórico}

Existen tres tipos de líneas de estudio en lo referente a la relación entre la migración y la salud mental de quien migra.

En el primer tipo se encuentran los concernientes a patologías mentales, enfocándose en la valoración de trastornos depresivos, conductuales y del aprendizaje, así como la evaluación del autoestima de los migrantes (Carlson y Jané, 2001).

Un segundo grupo de estudios es el que analiza la migración bajo la óptica del choque cultural que se produce entre la cultura original y la cultura anfitriona; con esto asumimos que con la migración, los implicados se enfrentan a un proceso de aculturación, durante el cual pueden contraponerse algunos aspectos culturales como su estilo de vida, modo de pensar, normas morales, valores, metas, entre otros, lo que en su conjunto compromete sus identidades y puede acarrearles ciertos problemas de salud (Gonzáles, 2006).

Un tercer tipo de estudio es el que entiende a la migración como un proceso psicosocial de duelo, condición a la que se ve expuesto todo migrante, considerando que las pérdidas migratorias son inherentes al desarraigo aun cuando esta situación fluctúa dependiendo de las variables relacionadas al proceso de pérdida: tipo de migración; distancia cultural; restricción al libre 
tránsito, tolerancia social y diversidad cultural de la comunidad anfitriona ; si es migración individual o colectiva; de la etapa del ciclo vital y migratorio, entre otros. Por lo tanto, la migración "per se" no produce ningún incremento en las probabilidades de tener algún trastorno mental, sino que depende de las experiencias traumáticas vividas durante el proceso, de la magnitud de los estresores y el tiempo de exposición a éstos (García y Sanz, 2002).

Boss (2001), en uno de sus estudios clínicos sobre pérdida ambigua, trabajó con pacientes inmigrantes y reporta que la nostalgia pasa a formar parte de la cultura familiar en el caso de comunidades de inmigrantes; quienes en reflexiones se preguntan de manera continua sobre el origen y composición de su familia y el espacio de identidad y pertenencia. En una migración, cada miembro de la familia la experimenta de una manera distinta de acuerdo a su edad y sexo, siendo para algunos mas sencilla de sobrellevar que para otros. En el caso de los niños y niñas migrantes, aún cuando pueden percibir su viaje al norte como una aventura, también tienen que lidiar con algunas pérdidas trascendentales y situaciones riesgosas.

Cuando hablamos de que la migración implica varios tipos de pérdidas, nos referimos a la característica de multiplicidad que poseen las pérdidas migratorias, es decir, con la mudanza se dejan muchas cosas valiosas para el migrante, y en ocasiones de una sola vez. Al movilizarse de una comunidad a otra, se viven pérdidas afectivas de familia, amigos y demás personas significativas que se quedan en el lugar de origen; pérdida por la lengua, y toda la simbología particular que ésta implica; pérdidas de prácticas culturales cotidianas como, hábitos, valores, costumbres, rituales y tradiciones que tienen que ser sustituidos por otros, o carecen de los medios para darles continuidad aun cuando así lo desean, como el obstáculo que para ellos representa el no poder disponer de algunos materiales necesarios para elaborar sus artesanías; pérdidas de estatus o nivel social; pérdidas de referentes físicos y ecológicos con los que a su vez tenían lazos ancestrales, como es el caso de algunos paisajes con cierta luminosidad, colores, olores, entre otros; situación que se complica por los riesgos físicos de la migración y el derrumbe de su proyecto migratorio (Falicov, 2001; Gonzáles, 2006; Martín, 2006).

Para Carbonell, Codina, Salas y cols. (2006) el duelo migratorio no elaborado tiene como consecuencia ciertos síntomas que pueden convertirse en un factor de riesgo a la salud mental, e incluso física, del migrante y su familia. Es debido a ello que se afirma que la migración tiene su costo social y psicológico, y entre los factores mas importantes se encuentran el estrés psicosocial relacionado generalmente con trastornos mentales y conductuales (García y Sanz, 2002; Maldonado, 2005). Todo esto, producto del agotamiento 
físico y mental que provoca la exposición prolongada a la pérdida ambigua; con un posible duelo complicado por causas ajenas a patologías internas (Boss, 2001)

Entre los estresores comúnmente hallados en el contexto de la migración se encuentran, la soledad, el fracaso del proyecto migratorio, la lucha por la subsistencia, el miedo a los peligros físicos, entre otros, siendo éstos la base psicosocial del síndrome de Ulises. En cuanto a la sintomatología, es muy variada y va desde síntomas depresivos como la tristeza, llanto, culpa e ideación suicida; síntomas ansiosos como el nerviosismo, las preocupaciones recurrentes y excesivas, irritabilidad, e insomnio; somatizaciones como cefalea, fatiga, problemas osteomusculares; así como problemas en el área confusional, desorientación espacial y temporal, fallas en la memoria, dificultades en las funciones de atención y concentración, entre otros (Achotegui, 2006; Giorguli, 2004; Guevara y Porras, 2007). El objetivo del estudio es conocer cuáles son los riesgos y vulnerabilidades de los niños y niñas migrantes que se movilizan al noroeste de la república Mexicana en búsqueda de trabajo, y de qué manera amenazan estos su salud mental.

\section{Metodología}

\section{Participantes}

El tamaño de la muestra fue de 358 niños debido a la necesidad de tener al menos 5 participantes por cada reactivo para fines de validación de las escalas. Por otro lado, 150 niños originarios de la sierra de Guerrero, vecinos y amigos de los entrevistados en Sonora.

El trabajo de campo para nuestra investigación se llevó a cabo en 16 localidades y campos agrícolas de Sonora y 5 localidades de la zona serrana de Guerrero. De las 16 localidades se estudiaron niños de la sierra de Guerrero particularmente del Municipio de Acatlán y sus alrededores, ubicando primero a los niños entrevistados en Sonora y después eligiendo a sus pares no migrantes en sus pueblos de origen.

En cuanto a los participantes de estudio se eligieron menores migrantes en edad escolar, de ambos sexos, itinerantes y pendulares, así como asentados y migrantes de segunda generación; alfabetizados al español; con edades entre los 8 y 14 años, con un promedio de 10.6 años de edad y una desviación estándar de 1.5; originarios del Estado de Guerrero en la República Mexicana. Por otro lado, en el municipio de Acatlán en el Estado de Guerrero se seleccionaron a niños con características similares sociodemográficas, ingreso, número de hijos, educación de la madre, que fueran vecinos o vivieran en la misma colonia o barrio pero que no presentarán ningún episodio migratorio 


\section{Medidas}

1. La Escala de Ansiedad Manifiesta en Niños Revisada (CMAS-R) elaborada por Reynolds y Richmond en Estados Unidos en el año de 1985; y traducida en 1997 por la Editorial Manual Moderno en México. Está compuesta de 37 reactivos de respuesta dicotómica (Si/No) mediante los cuales se obtiene una puntuación de ansiedad total, dividida en cuatro subescalas: ansiedad fisiológica, inquietud/hipersensibilidad, preocupaciones, sociales/ concentración.

2. El Cuestionario de Depresión para Niños (CDS) diseñado por Lang y Tisher en 1978 en Australia y traducido al castellano en España en el año de 1983, consta de 66 reactivos agrupados en 8 dimensiones, las que a su vez están agrupadas en 2 subescalas: Respuesta afectiva, problemas sociales, autoestima, preocupación por la muerte/salud, sentimiento de culpabilidad y depresión

Para las respuestas originalmente se utiliza una escala Likert de 5 puntos con rango de respuesta desde muy en desacuerdo a muy de acuerdo, sin embargo para la actual investigación solo se dejaron tres opciones de respuesta (en desacuerdo, no estoy seguro, y de acuerdo).

3. El Inventario de Estrés Infantil (IEI) elaborado por Ramírez (2005) y Vega (2007) con población mexicana, diseñado originalmente por 63 ítems con 6 opciones de respuesta en escala Likert que van de muy contento a muy nervioso; pasando por poco contento, nada contento, nada nervioso, y poco nervioso. Éste formato fue reducido a una versión mas corta de 30 reactivos con solo las 3 opciones de respuesta que corresponden a 3 niveles de estrés: Nada nervioso, moderadamente nervioso y muy nervioso; con dos secciones de respuestas, la primera sección para una respuesta dicotómica destinada a corroborar la ocurrencia del evento que se señala en la frase; y la segunda con una escala Likert para averiguar su intensidad. Para efectos del presente estudio se combinaron ambas versiones y se sustituyeron, de la primera versión, algunos reactivos que se consideró que estaban mas claramente redactados en la segunda. En cuanto a las opciones de respuesta, solo se dejo la escala Likert de 3 puntos que va de; nada nervioso a muy nervioso, esquematizándose en la hoja de respuestas con un icono emocional que denota nerviosismo/estrés.

\section{Resultados}

Primeramente, para las tres escalas utilizadas (CMAS-R, CDS e IEI) se realizó la prueba de Kaiser-Meyer-Olkin (KMO) y la prueba de esfericidad de Bartlett (Anderson, 2003). A partir del análisis factorial, el número de 
reactivos por factor se modificó con respecto a las dimensiones originales de estas escalas, simplificando en mayor medida el número de factores extraídos siguiendo el principio de parsimonia. Esto respondió al hecho de que en su versión original las escalas fueron validadas con poblaciones de perfil disímil a las del presente estudio.

La siguiente tabla muestra, además de las dimensiones tal y como fueron reestructuradas; las puntuaciones de alfa, varianza y número de reactivos por factor; así como las puntuaciones de KMO, Barlett, alfa y varianza totales correspondientes a cada uno de los tres instrumentos.

Tabla 2: Kaiser-Meyer-Olkin, test de Barlett, varianza y alpha por factores para la escala de ansiedad, cuestionario de depresión para niños e inventario de estrés infantil

\begin{tabular}{lcccccc}
\hline \multicolumn{1}{c}{ Factor } & Varianza & $\begin{array}{c}\mathbf{N}^{\mathbf{0}} \mathbf{d e} \\
\text { reactivos }\end{array}$ & Alpha & Media & DS \\
\hline Inquietud - Hipersensibilidad & 14.23 & 9 & .59 & .66 & .47 \\
Ansiedad fisiológica-fobias & 6.91 & 11 & .65 & .54 & .45 \\
Preocupasiones sociales-somatización & 5.55 & 8 & .62 & .42 & .49 \\
Escala de Ansiedad. KMO=.73/Barlet.00 & V.E. Total $=26.7 /$ Alpha & Total=.76 & & \\
Preocupación por la muerte-autoestima & 12.71 & 14 & .77 & 2.75 & 1.69 \\
Ánimo-Alegría & 7.22 & 13 & .75 & 2.14 & 1.51 \\
Sentimiento de culpabilidad & 4.89 & 11 & .71 & 3.38 & 1.63 \\
Problemas sociales-aislamiento & 3.18 & 11 & .67 & 2.74 & 1.67 \\
Cuestionario de depresión. KMO=.79/Barlett=.000/V.E. Total=28.01/Alpha Total=.84 \\
Estresores varios & 22.17 & 27 & .88 & 5.48 & .61 \\
Juego-Socialización & 4.41 & 20 & .89 & 5.18 & .73 \\
Pérdidas afectivas -migración & 3.52 & 15 & .83 & 5.11 & .75 \\
Competitividad & 2.97 & 10 & .73 & 5.17 & .75 \\
Inventario de estrés infantil. KMO=.90/ Barlett .000/ V.E. Total=33.09/Alpha Total=.94 \\
\hline
\end{tabular}

Fuente: Elaboración propia de los autores.

Los criterios que se siguieron para la exclusión de reactivos posterior al análisis factorial fueron: 1) Que en el contraste $t$ de student para el primero y cuarto cuartil de la medida no existiera diferencia significativa, lo cual indica que el reactivo no discrimina; 2) Que poseyeran un peso factorial menor de 0.35 ; 3) Que se encontraran alejados teóricamente de los nuevos factores encontrados; 4) Que mejoraran el valor de alfa si el factor era borrado de la lista; 5) Que generaran factores con muy poca varianza.

En los resultados podemos observar que las medias para las puntuaciones totales de estrés, depresión y ansiedad se encuentran dentro del rango de puntuaciones saludables. Sin embargo el $41.34 \%$ de los menores encuestados 
presentaba altas puntuaciones de estrés, el $20.4 \%$ altas puntuaciones de ansiedad, y el $10.6 \%$ altas puntuaciones de depresión.

Por otro lado, se encontró que los menores que presentaban altos niveles de estrés eran los migrantes que habían cambiado su residencia dos o más veces, a diferencia de los bajos niveles que presentaban los asentados o quienes tenían mas de 7 años viviendo en el mismo campo. La ansiedad, por otro lado, resultó ser mayor (al igual que el estrés) para los menores que tenían mayor numero de migraciones.

En la Tabla 3, se presenta una muestra constituida de 508 menores migrantes, la media de ansiedad total para los niños es de 14.32 y la desviación estándar de 4.81. Por su parte, las mujeres obtuvieron una puntuación de 16.20 para la media, así como de 5.03 para la desviación estándar para el factor ansiedad total. De esta forma, podemos decir que ambas puntuaciones de media caen dentro del sexto decil que comprende del 14.1 al 16.8 (dentro de éste se encuentra también la media general), el cual corresponde a las puntuaciones saludables. No existe diferencia significativa entre hombres y mujeres en el caso de esta variable $(T=.308 ; \mathrm{p}=.72)$

Por otro lado, la media para los hablantes de lengua indígena es de 14.72 y la desviación estándar de 3.88 para el Factor Ansiedad Total. En cambio, la media para los no hablantes es de 15.24 y la desviación de 5.17. Ambas medias situadas en puntuaciones saludables (sexto decil). Existe una diferencia significativa $(\mathrm{t}=.702 ; \mathrm{p}=.03)$; lo que habla de que la condición lingüística de los participantes está relacionada con el porcentaje de ansiedad total, aun cuando no hay evidencia que sea una relación causal. En este punto se vuelve necesario tomar en cuenta que para el caso de los hablantes la dispersión de las puntuaciones totales de los participantes es considerablemente menor que para el caso de los no hablantes,

Para depresión el caso de los hombres, la media fue de 101.23 y la desviación estándar de 22.69, ésta se ubica compartiendo el quinto decil (decil que entra dentro del rango saludable y que comprende del 93.7 al 108) con la media general.

Por su parte, las mujeres obtuvieron una puntuación de 108.99 para la media y 24.05 para la desviación estándar, ubicándose en un decil ulterior que la de los varones, cayendo de igual modo dentro del rango de puntuaciones saludables (79.3 a 136.8).

La media para los hablantes de lengua indígena es de 105.21 y la desviación estándar de 20.41 para el Factor Total Depresivo. En cambio, la media para los no hablantes $(n=280)$ es de 104.61 y 24.18 para el caso de la desviación estándar. Colocándose ambas en el quinto decil $(\mathrm{T}=.17 ; \mathrm{p}=.05)$. 


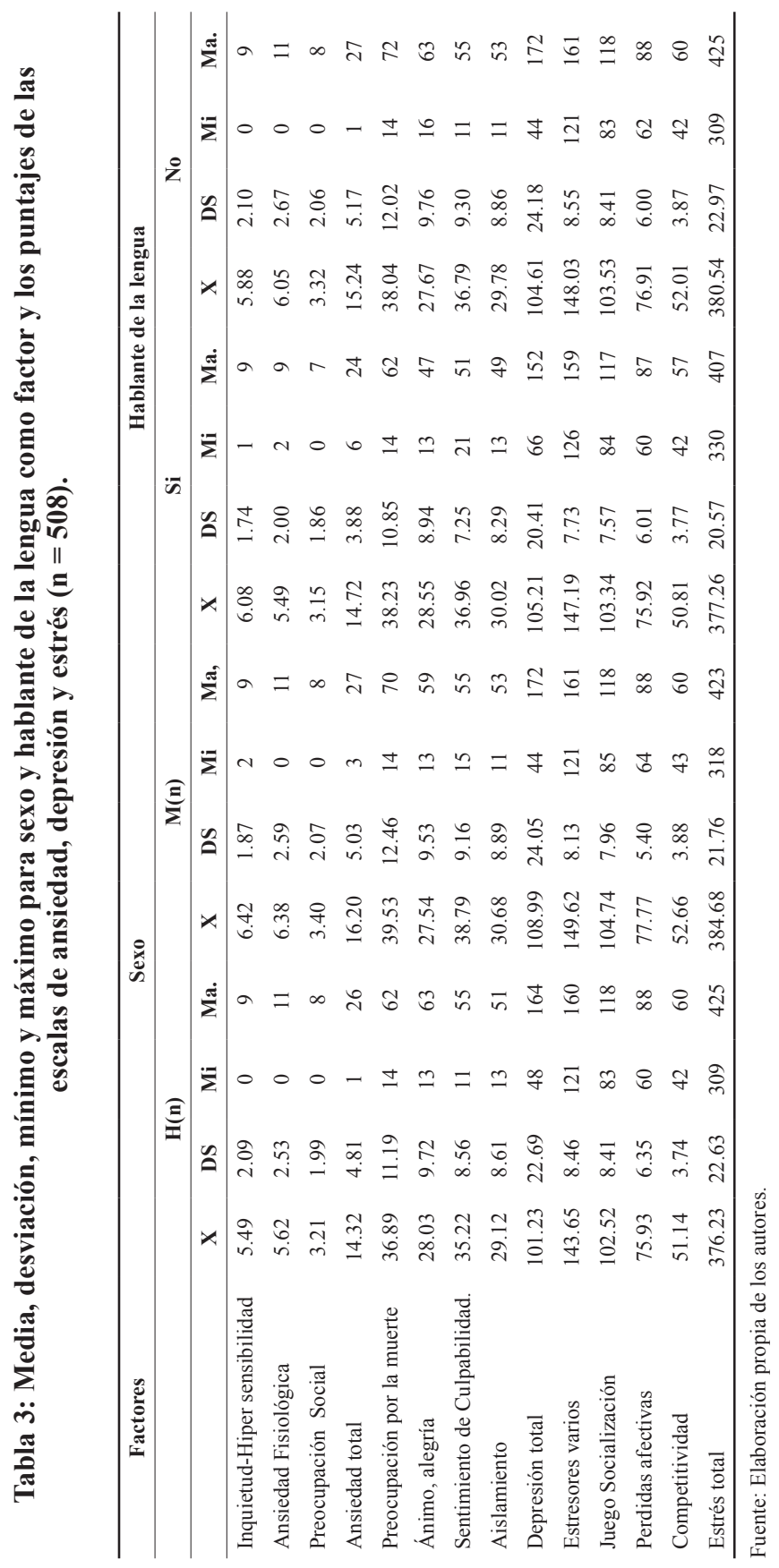


En estrés total para los hombres, la media fue de 376.23 y la desviación estándar de 22.66. Por otro lado, las mujeres obtuvieron una puntuación de 384.68 para la media y 21.76 para la desviación estándar.

Tanto la media para hombres como para mujeres se encuentra en el séptimo decil (376.2 al 389.6), que aun cuando es considerado saludable, debe tomarse en cuenta que está en el límite con respecto a las puntuaciones altas de estrés.

En la Tabla 4 los migrantes de primera generación (desde el nacimiento con sus padres; $n=213)$, la media de la puntuación natural de estrés fue de 381.57 (nuevamente dentro del decil 7) y la desviación estándar de 21.84.

Por otro lado, para los asentados y para los migrantes de segunda generación (desde los 13 años de edad hijos de padres migrantes), la puntuación para la media de estrés fue de 369.69 (decil 6, saludable) y 25.32 para la desviación estándar $(\mathrm{t}=4.07 ; \mathrm{p}=.01)$.

Las medias de ansiedad y depresión fueron siempre mayores en los niños migrantes que en los niños asentados. En las diferentes subescalas permanece la diferencia siendo mayores las medias para niños migrantes observando los mínimos y máximos los valores de media y de desviación estándar se encuentran siempre en los deciles saludables y las diferencias entre los grupos es significativas para el grado escolar, el tercer grado presenta siempre las medias más altas tanto en los totales como en las diferentes subescalas comparado con el quinto grado los valores de depresión y ansiedad se encuentran siempre en los deciles saludables.

En la Tabla 5 se llevo a cabo un análisis Múltiples Cruzados para Sexo, Grado Escolar, Condición Lingüística, Estado Migratorio, Ansiedad Total (Baja y Alta), Total Depresivo (Bajo y Alto) y Estrés Total (Bajo y Alto). Con respecto al proceso llevado a cabo para desarrollar este tipo de análisis en particular, cabe mencionar que para las variables dependientes (filas) se seleccionaron los factores Ansiedad Total, Total Depresivo y Estrés total; contrastando sus respectivos deciles bajos $(1,2$ y 3$)$ y altos $(8,9$ y 10). En cuanto a las independientes (columnas), se eligió únicamente una y es la correspondiente al Estado Migratorio (Migrante/Asentado).

Por último se incluyó como covariantes (estratos o capas) las variables Sexo y Condición Lingüística. Dentro de los hallazgos está el hecho de que aun cuando el $54.7 \%$ de la población era de varones y el $45.2 \%$, de las personas que puntearon con ansiedad alta eran mujeres y sólo $34.9 \%$ eran hombres. Lo opuesto sucedió con las puntuaciones bajas ya que de éstas 19 (el 61.2\%) eran hombres y 12 (el 38.7\%) eran mujeres; invirtiéndose la relación de puntuaciones altas a bajas. 


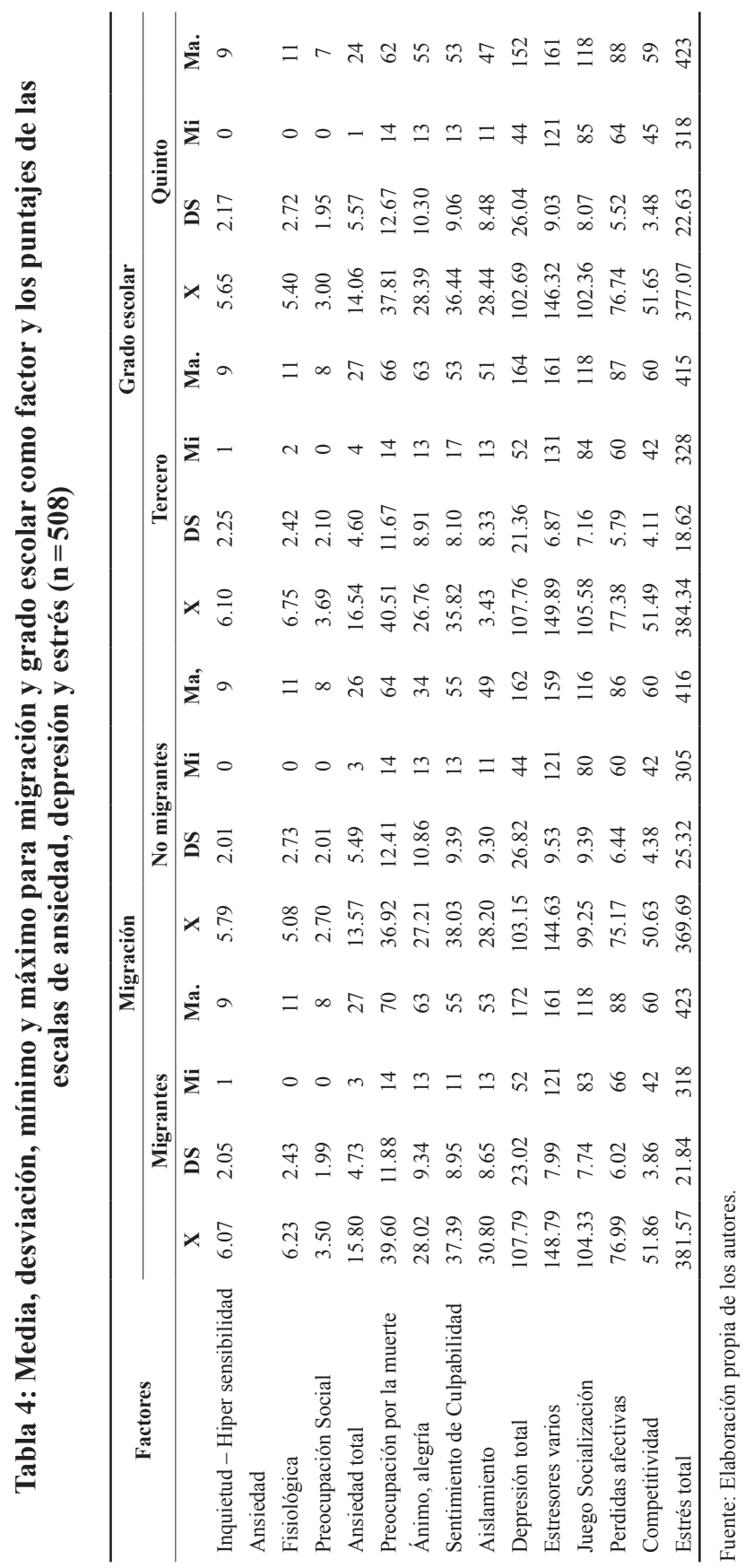




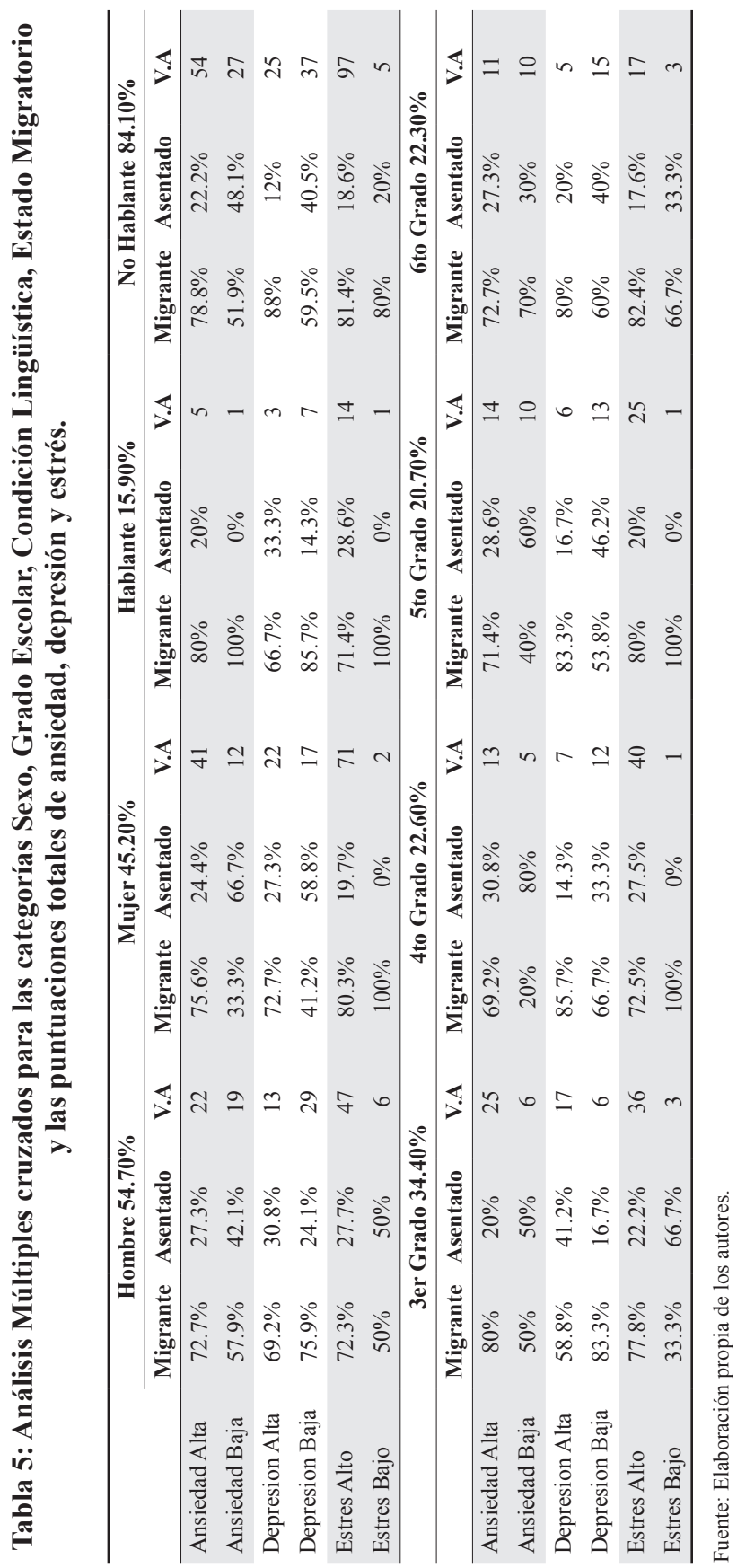


En cuanto a los puntajes altos totales de depresión, 22 (el 62.8\%) eran mujeres y 13 (el 37.1\%) hombres. Por su parte con las puntuaciones bajas ocurrió lo mismo que con la ansiedad, invirtiéndose la relación a 29 hombres (63\%) y 17 mujeres (36\%).

En las puntuaciones de estrés encontramos que el 71 de las personas con Estrés alto eran mujeres (60.1\%) y solo 47 (el 39.8\%) eran hombres (situación que no puede compararse con las puntuaciones bajas por carencia de participantes en este rango).

\section{Comparación por número de veces migrando y tiempo de asentado}

Los rangos que se elaboraron para esta ocasión fueron: el primero, para los que migrantes de segunda generación que nunca han viajado con su familia pero son hijos de migrantes no naturales del territorio $(n=87)$; el segundo, para las personas que han migrado 1 vez $(n=57)$, el tercero para quienes han migrado 2 veces $(n=48)$, el cuarto para los que tienen 3 migraciones acumuladas $(\mathrm{n}=36)$, y el quinto para los que tienen 4 o mas movilizaciones $(n=72)$.

Al llevar a cabo un análisis de varianza con la prueba One-way Anova, encontramos que existe una diferencia significativa por el número de migraciones realizadas para la variable Ansiedad Total $(p=3.87 ; p=.004)$, la prueba post-hoc (scheffee) indica que la diferencia está referenciada con la comparación entre los no migrantes (13.57) y el rango de los que han migrado 4 o más veces (16.58).

Por su parte, al realizar este análisis con la variable Estrés Total $(\mathrm{F}=4.86$; $\mathrm{p}=.001$ ), siendo los mismos rangos los que están generando este puntaje los que han migrado 2 veces, o de "4 o mas", con respecto a los que no han migrado.

A diferencia de las dos puntuaciones totales, al analizar el Total Depresivo no se encontró una diferencia significativa.

Después de realizar un análisis de varianza, podemos afirmar que existe una diferencia significativa para la cantidad del tiempo que tiene el menor en el campo relacionada a la variable Estrés Total $(\mathrm{F}=13.9 ; \mathrm{p}=.000)$, son los asentados hijos de migrantes los que presentan los más bajos niveles de estrés. La relación obtenida indica que a mayor tiempo de asentamiento, menor estrés

\section{Discusión}

De los datos obtenidos, la medida de estrés parece ser la más sensible a las comparaciones dentro y entre los grupos de migrantes y asentados. 
Las variables de $\mathrm{t}$ y sus medias son en todos los casos las de mayor valor numérico, le sigue ansiedad posteriormente sus dos subescalas de inquietud y preocupación social y finalmente depresión con ánimo-alegría y aislamiento.

En estas subescalas las medias más altas son para niños y niñas que no hablan la lengua original; los más pequeños de 8 a 10 años y los de quinto grado pertenecientes todos al grupo de migrantes. Los valores de t más altos corresponden a las comparaciones migrante-no migrante y hablante- no hablante de la lengua.

Podemos con certeza indicar que los niños migrantes puntualmente los más pequeños se encuentran con niveles de estrés más altos que los de los no migrantes y este proceso afecta su estado de ánimo y tranquilidad. En esta condición de migrante ser no hablante y niños más que el grado escolar y la edad parecen ser variables indicativas de mayor estrés.

De los modelos y teorías que la psicología ha desarrollado para estudiar este fenómeno podemos decir que, resaltan algunos como el modelo de Migración y Salud propuesto por Bhugra (2004), o la teoría del Síndrome de Ulises desarrollada por Achotegui (2006). Sin embargo, estos han sido creados bajo realidades desiguales como la migración internacional, o los desplazamientos permanentes (con asentamientos). Es por esto que no son del todo adecuados para el estudio de la migración interna y temporal (golondrina o pendular), como lo es el caso de la mayoría de los actores que participaron en el presente estudio.

En cuanto a las consecuencias psicosociales de este fenómeno, dependerán en gran medida de las características particulares de sus actores, de sus vulnerabilidades y resiliencias, de las circunstancias en que se da la situación, de la magnitud de los estresores y su tiempo de exposición a estos; ya que las secuelas de la migración per se no implican un cuadro psicopatológico, ni la desestructuración o desintegración de sus familias.

Los costos para una persona que migra de manera temporal no serán los mismos que las consecuencias para quien migra de manera permanente; así tampoco para quien migra solo, o quién lo hace en compañía de su familia y seres queridos; lo haga de manera voluntaria, o presionado por circunstancias religiosas, económicas, sociales y/o políticas; para quien llega a una comunidad que lo recibe con los brazos abiertos, o quien es recibido con el yugo de la discriminación.

El enfoque adoptado para la investigación en cuanto a la relación entre Migración/Salud Mental es el del duelo migratorio (Boss, 2001); el cual es puesto en duda por algunos estudios que puntualizan la relativización del desarraigo y la ruptura provocados por la nueva percepción del tiempo y el 
espacio asociada a la tecnología en comunicaciones (la que en determinado momento serviría como recurso psicosocial). La cual puede ser una explicación alternativa para el mantenimiento de una salud mental positiva para la muestra de menores migrantes en este estudio.

Por otro lado, el duelo también está vinculado a lo férrea y sistemática que fuera la rutina o cotidianeidad de las personas en cuestión. Cuánto más metódica fuera la rutina en sus lugares de origen, mayor debería ser la dificultad para sobrellevar el duelo en sus lugares de recepción.

Para la variable estado migratorio se realizaron correlaciones con las tres puntuaciones totales pero solo resultó significativa para estrés total. Este punto podemos explicarlo si pensamos en el estrés como una situación en la que existen mayores demandas ambientales de las que la persona percibe puede resolver satisfactoriamente, es decir, que excede sus recursos. Sin embargo el estrés no es una enfermedad como tal, sino es una condición reactiva que no ha trascendido a una patología del estado de ánimo, depresión o a una ansiedad (Hernández, 2008; Torres, Villoro y Ramírez, 2002).

Por su parte, en los análisis múltiples cruzados se pone a discusión el hecho de que las altas puntuaciones de ansiedad, depresión y estrés están siendo representadas en su mayoría por población migrante itinerante y no por asentados, situación que se hace notar aun cuando el $71 \%$ de la muestra este constituida por migrantes y el $19 \%$ por asentados. Esto pudo ser corroborado con un análisis de varianza de la correlación entre ansiedad total por número de migraciones, la que resultó ser significativa; situación que se repitió cuando esta variable fue correlacionada con el puntaje de estrés total (del mismo modo que ocurrió para el caso de la variable "tiempo viviendo en el campo" con este último).

Esta misma relación entre el tiempo de vivir en el campo agrícola y mayores niveles de estrés, ya ha sido registrada en otros estudios acerca del conocimiento de las características psicosociales de los migrantes internos dirigidos hacia territorio estatal. La explicación que se ha dado al respecto, es que los migrantes que recién se han asentado viven situaciones estresantes que enfrentan de manera emocional, particularmente discriminación, exclusión y agresión física y verbal (Laborín, 2008).

Otro de los hallazgos de este análisis son las puntuaciones mayores de ansiedad, estrés y depresión para el caso de las mujeres con respecto a los hombres, en ocasiones inclusive duplicándolos en número. Estudios anteriores (Vera, 1996), han demostrado que la mujer tiene mayor permisibilidad por parte de la sociedad para reconocer en público las emociones que pudieran estar socialmente relacionadas con la vulnerabilidad, como lo son las asociadas a la depresión. Dicho de otra manera, el estar deprimido mina de algún modo 
la autosuficiencia que se supone debería caracterizar a los varones, lo que implicaría que pudiera ser considerado por los demás como señal de debilidad (Bonino, 2000).

\section{Conclusión}

Podemos concluir, que los altos índices de estrés que presentan el $41.34 \%$ de los participantes, pueden ser relacionados a los estragos ocasionados ante las constantes migraciones que viven estos menores. Dicho de otra manera, se pueden asociar a la imposibilidad que establece la situación del migrante pendular o golondrino para resolver favorablemente el duelo que las pérdidas migratorias suponen, al no terminar de establecerse en un solo lugar. Esta realidad es distinta para los migrantes permanentes, asentados en nuevas localidades geográficas, quienes viven un proceso lineal y no circular, facilitándoles la resolución saludable del duelo.

En cuanto a la naturaleza de estos estresores, se encontraron algunas dificultades propias de climas desérticos, a los que estos niños y niñas no estaban acostumbrados en sus lugares de origen y ahora tienen que soportar en su nuevo domicilio, trabajando horas enteras bajo el sol abrasante y el polvo que fácilmente es levantado por el viento. Otro de los estresores psicosociales que manifiestan estos menores es el referente a las largas jornadas de trabajo a las que tienen que someterse, lo cual esta directamente relacionado con el estresor anterior. Un tercer estresor está enlazado a las preocupaciones que les despiertan los riesgos de la trayectoria migratoria, ligada a historias de accidentes que han oído o vivido.

Es necesario recordar que son estos estresores los que en su magnitud o intensidad estarían incidiendo en una desfavorable resolución del duelo migratorio y por consiguiente en la presencia de una salud mental negativa en lo que a estos menores migrantes respecta.

Si observamos la tabla 6 podemos percatarnos de que 20 de los 508 participantes $(4.0 \%)$ presentaron la sintomatología completa del duelo migratorio (estrés, ansiedad y depresión), situación que los coloca del lado negativo del continuo salud mental - enfermedad (deciles 8, 9 y 10). Por el contrario, solamente 3 participantes $(0 \%)$ se encontraba dentro del rango de salud mental positiva (deciles 1\%, 2\%, 3\% y 5\%).

Tabla 6: Caracterización de la Población por Deciles Extremos

\begin{tabular}{cccccccc}
\hline Deciles & Participantes & Varones & Mujeres & Edad $(\mathbf{X})$ & Grado $(\mathbf{X})$ & Lenguas & Migraciones $(\mathbf{X})$ \\
\hline 1,2 y 3 & 3 & 2 & 1 & 12.3 & 6 & Nahuatl & 1 \\
8,9 y 10 & 20 & 5 & 15 & 10.3 & 4.1 & Nahuatl, Zapoteco & 3 \\
\hline
\end{tabular}

Fuente: Elaboración propia de los autores. 
Partiendo de esta caracterización, dentro de los factores de riesgo que se presume, pudieran estar inclinando la balanza hacia una salud mental negativa, el trabajo infantil y el mal clima al que son expuestos estos niños y niñas durante y fuera de estas jornadas laborales; factores que ya fueron mencionados previamente como los estresores principales.

Por otra parte, en cuanto a los recursos disponibles por estos menores que se hicieron presentes en la investigación de campo, podemos resaltar a las redes de apoyo y la tendencia al juego (factores de protección). Ambos recursos le sirven al menor para mantener una salud mental positiva y lo resguardan de los efectos del conflicto y la frustración. Es el comportamiento lúdico, sumado a un número menor de riesgos potenciales durante el trayecto migratorio (a diferencia de la literatura acerca del migrante internacional indocumentado), lo que contribuye a que el cuadro sintomático no tenga proporciones patológicas.

\section{Referencias}

ACHOTEGUI, J. Estrés límite y salud mental: El síndrome del inmigrante con estrés crónico y múltiple (Síndrome de Ulises). Barcelona: Universidad de Barcelona, 2006. p. 59-85.

ANDERSON, Hair. Análisis multivariante. España: Pearson, 2009.

BONINO, L. Varones, género y salud mental. Desconstruyendo la "normalidad" masculina. Nuevas visiones de la masculinidad. Barcelona: Icaria, 2000.

BOSS, P. La pérdida ambigua. España: Editorial Gedisa, 2001.

CARBONELL, T.; CODINA, B.; SALA, S.; GONFAUS, M.; PÉREZ, N.; TORRALBA, V. El duelo de los inmigrantes (Proyecto de Intervención Grupal). Congreso de Escuelas Universitarias de Trabajo Social: Ponencias y Comunicaciones, VI., Escuela Universitaria de Estudios Sociales, Universidad de Zaragoza, España, 2006. p. 1-14.

BHUGRA, D. Migration and Mental Health. Acta Psychiatrica Escandinavica, v. 109, n. 4, p. 243-258, 2004.

CARLSON, J.; JANÉ, M. Salud mental infantojuvenil en inmigrantes. Barcelona: Universidad Autónoma de Barcelona. In: Psiquiatria.com, p. 1-3, 2001.

COS, F. Sirviendo a las mesas del mundo: los niños y niñas jornaleras agrícolas de México. In: DEL RÍO, N. (coord.). La infancia vulnerable de México en un mundo globalizado. México: UAM-UNICEF, 2000. p. 79-84.

Dif - Sonora. Situación de menores migrantes hijos de jornaleros agrícolas migrantes. Estudio realizado en colaboración con INEGI, INEA, CONAFE, UNISON y el Programa de Jornaleros Agrícolas. 1997.

FALICOV. Migración, pérdida ambigua y rituales. [Online] Perspectivas Sistémicas. La Nueva Comunicación, 20001. [Trabajo presentado en: Congreso Nacional de Terapia Familiar de la Asociación Mexicana de Terapia Familiar, VIII., y en: Conferencia CEFYP, Buenos Aires, 2001.] 
GARCÍA, J.; SANZ, C. Salud Mental en Inmigrantes: El nuevo desafío. Revista Medicina Clínica, Zaragoza, Universidad de Zaragoza, p. 137-191, 2002.

GIORGULI, S. ¿Juventudes truncadas? El impacto de la migración mexicana hacia EUA sobre los jóvenes que se van, los que se quedan y los hijos de los que se fueron. Congreso Mundial de Movimientos Humanos e Inmigración, Institut Europeu de la Mediterranea, 2004. p. 1-4.

GONZÁLES, V. El duelo migratorio. Revista Trabajo Social, Bogotá, n. 7, p. 77-97, 2006.

GUEVARA, L.; PORRAS, S. El sindrome de Ulises. Hospital General de Elda, España, 2007.

HERNÁNDEZ, E. El estrés infantil. Zona Pediátrica, Comunidad de Salud Infantil, 2008.

INEA. Programa estratégico de atención a jornaleros agrícolas migrantes. Trabajo presentado en: Foro Nacional para la Vida y el Trabajo, mayo 2007, México, DF.

INSTITUTO Nacional de Estadística Geografía e Informática. Censo general de población y vivienda, 1990. México, DF, 1995.

LABORÍN, J. Adaptación psicológica de migrantes indígenas asentados en el Estado de Sonora, México. Tesis (Doctor en Ciencias Sociales) - Universidad Autónoma de Sinaloa, Culiacán, Sinaloa, 2008.

LANG \& TISHER. Cuestionario de depresión para niños - manual. 5. ed. Traducido al español por TEA Ediciones. Madrid, 1997.

LARA, S. Características del empleo rural en el umbral del siglo XXI. In: Momento Económico - Revista electrónica bimestral, México: Instituto de Investigaciones Económicas, Ciudad Universitaria, UNAM, enero 1997.

LÓPEZ, M. Trabajo infantil y migración en el Valle de San Quintín, Baja California. Foro Invisibilidad y Consciencia: Migración interna de niños y niñas jornaleros agrícolas en México, septiembre, 2002, Universidad Autónoma Metropolitana/ UNICEF. p. 1-19.

MALDONADO, M. Los costos psicológicos de la migración. Revista La Familia, marzo 2005.

MARTÍN, C. Las migraciones externas desde un enfoque psicosocial: Familia y salud mental. Mesa Redonda: Congreso Regional de la Asociación Mundial de Psiquiatría; Congreso Panamericano de Salud Mental Infanto Juvenil, II.; Conferencia Internacional Hospital Psiquiátrico de la Habana - PSICOHABANA III., 2006. p. 1-7.

RAMIREZ, H. La resiliencia. [Online]. Disponible en: <www.geocities.com/ centrotecnicas/resiliencia.hatml>. Recuperado en: 5 oct. 2005.

REYNOLDS \& RICHMONDS. Escala de Ansiedad Manifiesta en Niños (Revisada). Manual. Traducido al español por Editorial El Manual Moderno. México, DF, 1997.

SEDESOL. Reglas de operación del programa Hábitat. México, 2006. 
TORRES, J.; VILLORO, R.; RAMÍREZ, T. La salud de la población indígena en México. Iniciativa Global de Equidad en Salud. Fundación Rockefeller. Fundación Mexicana para la Salud, 2002.

UNICEF. Jornaleros agrícolas y trabajo infantil. Información en línea UNICEF.ORG, 2007.

VEGA, Z. Primer estudio de validez de constructo del Inventario de Estrés Infantil (IEI). Revista Electrónica de Psicología Iztacala, v. 10, n. 3, p. 69-73, 2007.

VERA, J.A. Comparación de los síntomas de depresión en mujeres en dos poblaciones. Psicología y Salud, Instituto de Investigaciones Psicológicas de la Universidad Veracruzana, Nueva Época, v. 8, jul.-dic., p. 7-17, 1996.

VERA, J.; RODRÍGUEZ, C.; BORJA, B. Condiciones psicosociales de los niños jornaleros agrícolas migrantes. Revista Intercontinental de Psicología y Educación, v. 9, n. 1, p. 21-48, 2005. 